$\begin{array}{ll}\text { Italique } & \text { Italique } \\ \text { Poésie italienne de la Renaissance }\end{array}$

XV | 2012

Varia

\title{
Ariosto petrarchista: appunti sul sonetto «Aventuroso carcere soave»
}

Alberto Roncaccia

\section{(2) OpenEdition}

Journals

\section{Edizione digitale}

URL: http://journals.openedition.org/italique/350

DOI: 10.4000/italique.350

ISSN: 1663-4438

\section{Editore}

Librairie Droz

\section{Edizione cartacea}

Data di pubblicazione: 1 dicembre 2012

Paginazione: 149-161

ISBN: 978-2-600-01639-1

ISSN: 1423-3983

Notizia bibliografica digitale

Alberto Roncaccia, «Ariosto petrarchista: appunti sul sonetto «Aventuroso carcere soave» », Italique [Online], XV | 2012, online dal 01 décembre 2015, consultato il 19 avril 2019. URL : http:// journals.openedition.org/italique/350; DOI : 10.4000/italique.350 


\author{
Alberto Roncaccia \\ A R I O S T O P E T R A R C H I S T A: \\ A P PUNTI SUL SONETTO \\ «A VENTUROSO CARCERE SOAVE»
}





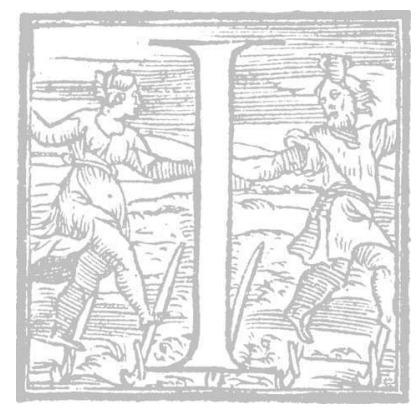

I sonetto Aventuroso carcere soave ha spesso suscitato l'attenzione degli studiosi e degli antologizzatori dell'Ariosto rimatore. L'interesse è pienamente giustificato dai diversi livelli di rappresentatività del componimento: produzione volgare dell'autore, petrarchismo del Cinquecento, lirica d'amore europea. ${ }^{\mathrm{I}}$ Come ricorda Giulio Ferroni, si tratta di «uno dei sonetti più celebrati dalla critica». ${ }^{2}$ Riconsiderato all' interno del canzoniere manoscritto - o progetto d'autore di un canzoniere - studiato da Cesare Bozzetti, il testo rivela con chiarezza la propria funzione di snodo logico-tematico.

Nel percorso macrotestuale, dopo una fase più convenzionale giocata sull' amore a distanza, il sonetto segna, a sorpresa, un passaggio al tema dell'appagamento sensuale del desiderio e dell'amore-passione. Il lessico cambia registro e l'intonazione si fa esplicitamente erotica ed elegiaca. A questo proposito, Stefano Carrai ha parlato di un «petrarchismo irrobustito dal connubio con generi e forme della tradizione classica e umanistica». ${ }^{4}$ Tale pratica, imitativa e allo stesso tempo contaminatoria, risulta già chiara anche senza tener conto del dato macrotestuale. Si può parlare quindi, a ragione, di «rivisitazione di esemplari petrarcheschi» con «esito volutamente straniante».5 In tale esito, nelle modalità di una calibrata infrazione tematica e formale, si colgono l'originalità scrittoria e l'autonomia critica del Ferrarese. Il procedimento che lo produce è stato indicato con precisione da Bigi e, più recentemente, confermato da Carrai come «creazione di un ritmo sintattico diverso da quello metrico». ${ }^{6}$ Questa chiave di lettura può essere verificata nei puntuali scarti lessicali e semantici operati nei confronti degli stilemi petrarchistici, o in circoscritti elementi di tono ironico o sfalsato. Alla scansione sintattica, infatti, sembra affidata una funzione misuratamente eversiva ${ }^{7}$ nei confronti della corrispondenza di temi e di forme che si affermava come indissociabile nella lirica contemporanea. All'altezza cronologica in cui l'Ariosto opera, va ricordato, il processo di modellizzazione del petrarchismo bembiano non era del tutto compiuto. ${ }^{8}$ E a livello di sintassi logico-tematica, secondo le indicazioni di Bigi e di Carrai, che possiamo cercare di verificare nel singolo componimento alcuni precisi momenti di scarto o di infrazione rispetto alle implicite 
attese 'di genere'. Citiamo il testo dalla trascrizione di Cesare Bozzetti: 9

Aventuroso carcere soave, dove né per furor, né per dispetto, ma per amor et per pietà distretto la bella et dolce mia nemica m'have, li altri prigioni al volger de la chiave s'attristano, io m'allegro, ché diletto et non martir, vita et non morte aspetto; né giudice sever né legge grave, ma benigne accoglienze, ma complessi licentiosi, ma parole sciolte da ogni fren, ma risi, vezzi et giuochi, ma dolci baci dolcemente impressi ben mille et mille et mille et mille volte; et se potran contarsi ancho fien pochi.

Il sonetto può essere considerato di tipo monoperiodale, in linea con Petrarca, che ne ba 4 (o secondo alcuni 5), e con Bembo, che ne ha una quindicina. Ariosto ne ba 607 , con una proporzione più prossima a quella bembiana se confrontata all'esiguità del corpus. Sulla base dell' inventario condotto da Soldani, gli esempi di sonetto monoperiodale precedenti a Petrarca risultano rarissimie non se nepresenta alcuno in Dante. ${ }^{\text {10 }}$

La sintassi monoperiodale, pur in sintonia con la sensibilità del petrarchismo del primo Cinquecento, favorisce tuttavia l'effetto di scarto tematico dato nelle terzine. Nonostante il sonetto si dia come un solo periodo, può essere sintatticamente assimilato ad un schema di costruzione $4^{+}+$I0. All'interno della campata periodale, la prima quartina e il resto del sonetto sono da classificare come due frasi principali coordinate. Tra le due frasi, infatti, non si rilevano elementi di connessione subordinante. L'unica connessione è data dall'incipitale vocativo prolettico, seguito però dal «dove» con valore di congiunzione subordinativa e, per questo, a livello sintattico piu strettamente integrato nella prima quartina. Lo stacco tra prima quartina e versi successivi è anche contrassegnato dalle forme verbali, rispettivamente al passato ("distretto [...] m'bave») e poi al presente e al futuro ("fien», v. I4).

In base a questa costruzione, alla prima quartina possiamo attribuire valore di 'proposta' e di sintesi tematica, di cui $i$ seguenti Io versi forniscono esplicazione e sviluppo. 
Il tema del carcere amoroso, ${ }^{\mathrm{II}}$ enunciato dal fluido e simmetrico vocativo del primo verso, dopo la breve sospensione creata dal nesso «dove», è dato come filo logico principale dal verbo al passato prossimo «distretto [...] m'have» posto in enjambement $e$ in inversione ai $v v .3-4 . \mathrm{Il}$ soggetto «la bella e dolce mia nemica», incastonato nell'enjambement del verbo composto, produce un effetto di dilatazione metrico-sintattica. Notiamo come l'innalzamento di tono, costruito attraverso usuali dispositivi rallentanti, culmini nel latinismo d'uso poetico della terza persona del verbo avere, «m'have». Il posizionamento a destra del verbo, che marca la forte e strutturante tmesi sintattica già rilevata, ne conferma la funzione di chiusura frastica.

Nei due versi centrali della prima quartina, la ripetizione correlativa del né e il ma oppositivo sorreggono l'espansione del vocativo, cosi da sospendere liricamente l'essenzialità enunciativa e nominale dei vv. I e 4, che delimitano la prima sequenza logica del componimento. Nella quartina, notiamo, il contrasto tra $i$ versi centrali e gli esterni è sottolineato stilisticamente dall'opposizione di rime in sillaba aperta e cbiusa. I vv. 2-3, inoltre, sono imbricati dal chiasmo nominale oppositivo furor dispetto + amor - pietà. Un sourachiasmo di nomi e aggettivi, di tipo assimilativo, è inoltre reperibile nei vv. I e 4 : carcere soave + bella e dolce mia nemica. La compiutezza architettonica del doppio chiasmo è sottolineata stilisticamente dal verbo have, non solo per la detta funzione sintattica, ma anche per la ripresa, come chiusura fonica, della sonorità che apre la quartina.

A livello tematico, la prima quartina propone una sequenza di tre elementi: 'piacevolezza del carcere amoroso', 'le ragioni che lo rendono tale', 'la dolcezza dell' amata'.

Questa proposta costruisce tematicamente la restante parte del sonetto:
v. I
piacevolezza del carcere amoroso
vv. 5-6
VV. 2-3
le ragioni che lo rendono tale
VV. 7-I I
V. 4
la dolcezza dell'amata
VV. I 2-I 4

Tale costruzione è verificata dalla ripresa dei connettori sintattici della prima quartina nella più lunga sequenza successiva.

$\begin{array}{llllll}\text { NEGATIVI: } & \text { né... né... } & \text { v. } 2 & \text { non... non... / né...né... } & \text { v. } 7 \text { e } 8 \\ \text { CAUSALI: } & \text { per... per... } & \text { vv. 2-3 } & \text { ché... } & & \text { v. } 6 \\ \text { AVVERSATIVI: } & \text { ma... } & \text { v. } 3 & \text { ma... } & \text { (cinque volte) } & \text { v. 9- I } 2\end{array}$


Una scansione sintattica di rilievo riguarda, inoltre, le due terzine, introdotte da «ma» e rette a livello logico da «aspetto» (v. 7). Di questo elemento verbale, le due terzine espandono il complemento diretto, espresso nella quartina dalla coppia «diletto» $e$ «vita». L'anafora del $\mathrm{ma}$, che qui diviene congiunzione enfatica, perdendo la prima funzione avversativa, crea un accumulo volto a visualizare in elementi concreti quanto nei versi precedenti veniva dato dai petrarcheschi amor $e$ vita. La correlazione del $\mathrm{ma}$, oltre a unire le due terzine, tende a limitare progressivamente, fino ad azzerarla, la possibilità di uno sviluppo logico-ragionativo, moraleggiante, di quanto detto nella seconda quartina, cosi da prolungare la dimensione esclamativa introdotta dal primo verso.

La griglia sintattica del sonetto sostiene i legami semantici individuabili nella progressione logica tra quartine e terzine. Nelle quartine, ad esempio, notiamo la ripresa semantica del «carcere» del v. I nei «prigioni» del v. 5. Successivamente, la coppia «furor» e «dispetto» del v. 2 viene poi amplificata in quella «martir» e «morte» del v. 7.

La corrispondenza sintattica tra il v. 3 e i vv. 9-I2, indicata dal ma, $\grave{e}$ stabilita a livello semantico dal campo semantico del serrare, stringere, espresso da «distretto» al v. 3 e ripreso da «complessi», «sciolte», «fren» nella prima terzina.

Il legame tra $v .4$ e seconda terzina è dato dalla ripresa del tema della dolcezza. Lo si vede nella quartina riferita petrarchisticamente all'amata ("dolce mia nemica») e nella seconda terzina, prima esplicitata in chiave sensuale ("dolci baci», etc.) e poi riespressa iperbolicamente nei vv. I3-I4. A livello fonico, il legame è confermato dall'insistenza ritmica della sillaba mi, ripetuta quattro volte nel v. I3 e già significativamente raddoppiata nell'espressione «mia nemica» (v. 4). A livello sintattico e semantico, lo schema compositivo permette, quindi, di cogliere la progressione complessiva del discorso: a partire da una prima quartina di intonazione ineccepibilmente petrarchistica, le figure si sviluppano in direzione di un amore passionale e terreno piuttosto che del convenzionale amore virtuoso e spirituale. In questo senso, l'immagine del carcere, di solito usata per identificare e mortificare la corporeità umana viene reinterpretata in chiave positiva ed edonistica. ${ }^{\mathrm{I}}$ Nelle terzine, il richiamo intertestuale all' autorità erotica catulliana va certo ritrovato nel numerale che riprende il famosissimo carme $V$ ( $~ \mathrm{Da}$ mi basia mille» etc.), dove abbiamo la reiterazione del «mille» riferito ai 
baci degli amanti, ma anche nei «rumores[...] senum severiorum», cui rinvia il $v$. 8 del sonetto, "né giudice sever né legge grave». Per il motivo del conteggio dei baci, possiamo ricordare anche il Catullo del carme VII, di cui si può citare la chiusura ottativa: «nec pernumerare curiosi / possint nec mala fascinare lingua». Anche i "giuochi» del v. II, non proprio petrarcheschi nell'uso al plurale, sono portatori di una connotazione erotica per $i$ multa iocosa, che indicano gli atti d'amore, di Catullo иІІ 6: «ibi illa multa tum iocosa fiebant». Segre e i commenti successivi ricordano, inoltre, il riferimento Properzio II I5, 50: "romnia si dederis oscula, pauca dabis».

Osservando nel lessico, quindi, il grado di rispetto dell'uso petrarchesco e bembiano, è evidente come lo scarto nei confronti dell'uso convenzionale si concentri nelle due terzine, accentuando l'effetto di separazione dalle quartine per via di espressioni come «complessi / licentiosi» $e$ «dolci baci dolcemente impressi». Queste forme sono messe in evidenza proprio per il contrasto creato con sequenze iper-petrarchesche, come l'espressione «la bella e dolce mia nemica», e per il processo di risemantizzazione a ritroso di stilemi canonici. La tradizionale pratica di recupero e di imitazione è cosi compromessa dall'interno, per effetto di sottrazione nei confronti dell'apparente garanzia di significato e di rispetto dei valori originali del modello.

In margine a questi rilievi compositivi, si coglie nella seconda quartina la possibile attivazione di un livello di senso metatestuale. L'opposizione tematica tra "gli altri prigioni» e l'《io», data in forma di dichiarazione di poetica, potrebbe essere intesa come riferimento non generico a Petrarca, in cui l'immagine del carcere o prigione ricorre, insieme al desiderio d'esserne liberato: "aprasi la pregione, ov'io son chiuso, / et che'l camino a tal vita mi serra» ( $\operatorname{Rvf} 70,2)$; "Ma'l sovrastar ne la pregion terrestra / cagion m'è, lasso, d'infiniti malì ( $\operatorname{Rvf} 86,5)$; "Fuggendo la pregione ove Amor m'ebbe / molt'anni a far di me quel ch'a lui parve» (Rvf 89, I-2); "chiuse 'l mio lume e 'l suo carcer terrestro» (Rvf 306, 4); "et da quel suo bel carcere terreno» (Rvf 325, IOI); "O felice quel di che, del terreno / carcere uscendo, lasci rotta et sparta / questa mia grave et frale et mortal gonna, I/ et da si folte tenebre mi parta» ( $\mathrm{Rvf}$ 349, 9-I2); "felicem animam terreno de carcere digressuram〉 (Senili XI I). ${ }^{\mathrm{I}}$

Oltre che con Petrarca, si può stabilire un legame di notevole pertinenza con il sonetto Io8 di Bembo, nei cui confronti Ludovico alimentava una 
amichevole polemica sulla concezione amorosa fin dal carme $\mathrm{Ad}$ Petrum Bembum, ${ }^{14}$ che si ritiene databile al 1498-99. Citiamo il sonetto bembiano nella versione stampata nel I530, dove è il numero 99, sottolineando alcuni elementi di riscontro testuale o tematico:

Tanto è l'assenzo e 'l fel ch'io rodo e suggo,

Ch'omai di lor mi pasco e mi nodrisco,

E son sì avezzo al foco, ond'io mi struggo,

Che volontariamente ardo e languisco.

E se del carcer tuo pur talor fuggo,

Per fuggir da la morte, e tanto ardisco,

Tosto ne piango, et a prigion rifuggo,

Amor, più dura, in pena del mio risco.

E fo come augellin, che s'affatica

Per uscir de la rete, ov'egli è colto;

Ma quanto più si scuote, e più s'intrica.

Tal fu mia stella il dì che nel bel volto

Mirai primier de l'aspra mia nemica,

Ch'a me tutt'altro e più me stesso ha tolto. ${ }^{\text {is }}$

Rispetto a Petrarca e a Bembo, in Ariosto è esplicita la valorizzazione del carcere, cioè del corpo terreno, e, con un vero e proprio capovolgimento di significato, del motivo del serrare, trasferito dalla «rete» del cacciatore $^{\mathrm{IO}}$ ai «complessi / licentiosi》 dell'amata. Diversi indicatori testuali, tra cui, in Bembo, l'espressione "raspra mia nemica», suggeriscono ancora una mirata presa di distanza dal petrarchismo moraleggiante.

Un valore metatestuale può essere attribuito, in questo senso, anche all'originale sintagma «parole sciolte» (v. Io). ${ }^{17}$ Le parole del sonetto, non solo quelle degli amanti, possono essere intese come sciolte, 'libere', proprio in forza della rivalorizzazione del carcere terreno. ${ }^{\mathrm{I}}$ All'interno di un medesimo modello di lingua e di stile, il cambiamento riguarda soprattutto i valori di riferimento. Il topos della dolcezza della prigione e delle catene amorose - a sua volta ripreso dalla lirica trobadorica - ̀̀ in ogni modo già in Petrarca, in particolare nel sonetto 89, Fuggendo la pregione ove Amor m'ebbe, la cui conclusione, nelle terzine, riconverte il topos in chiave moraleggiante e riafferma l'esigenza, pur tardiva, di liberarsi dalla 'prigione' amorosa: ${ }^{19}$ "Onde più volte sospirando indietro / dissi: Obimè, il giogo et 
le catene e i ceppi / eran più dolci che l'andare sciolto. / / Misero me, che tardo il mio mal seppi; / et con quanta faticha oggi mi spetro / de l'errore, ov'io stesso m'era involtoly ( $\operatorname{Rvf} 89$, 9-14). Anche nell'uso verbale, quindi, usando il presente e infine il futuro, Ariosto modifica il dato canonico che presenta la dolcezza del carcere amoroso come un «errore» del passato, pur riconoscendola maggiore di quella dell" «andare sciolto».

A questo punto, osservata e confermata nella struttura logico-tematica la pratica di allontanamento dal modello, vale forse la pena di non postulare come acquisito il rispetto dello standard metrico petrarchista. Come si è visto, le infrazioni si giocano a un livello minimo e, soprattutto per la distanza che ci separa dall'estetica imitativa rinascimentale, queste minime variazioni non possono forse essere colte del tutto dal nostro orecchio. Una ricognizione sistematica della scansione sintattica e metrica delle Rime di Ariosto potrebbe, credo, misurare e rendere conto degli eventuali microindicatori di variazione nei confronti del modello. La breve lettura di un singolo sonetto, evidentemente, non può che offrire uno spunto di riflessione, porre l'interrogativo. Notiamo, allora, che nel testo l'opzione d'insieme resta melodica e la partitura fonica è coadiuvata dalle frequenti correlazioni. Anche l'uso di ictus contigui, presente in due casi, risponde agli usi petrarcheschi e bembiani. ${ }^{20}$ Se, ad esempio, l'incontro di accenti di 4a-sa, che si rileva nel $v$. 7 , è forse meno abituale, da considerare tipico è piuttosto quello, in sinalefe, di 6a-7a nel v. I4, proprio in chiusura di componimento. ${ }^{2 \mathrm{I}}$ Piuttosto, è da notare il fatto che nello stesso verso siano in contiguità e senza sinalefe anche gli accenti di ga e I0a. Questa compresenza di ictus contigui in un verso conclusivo sembra confermare la pertinenza di una ricerca, anche a livello metrico, di lievi incrinature nei confronti delle pratiche consolidate.

A livello rimico, una sfumatissima infrazione può essere attribuita probabilmente alla rima giochi - pochi, al plurale non petrarchesca e non bembiana, messa in evidenza dall'essere la sola rima in sillaba aperta nelle terzine. ${ }^{22}$ Da segnalare, almeno come attacco duro, ${ }^{23}$ è la dialefe «da ogni», con cui inizia il v. II, per di più in enjambement con il verso precedente. Da notare, inoltre, come il primo e l'ultimo verso deroghino alla generale tendenza a costruire endecasillabi bipartiti (o bipartibili), presentando accenti di riferimento comuni, 2a-4a-6a, ed evitando la cesura, rispettivamente, grazie al termine sdrucciolo e alla sinalefe che combina due accenti forti. La cesura, in altro modo, cioè per 
effetto di accumulo, è evitata anche per il v. I3, che Roberto Fedi definisce di intonazione «madrigalesca e cantabile». ${ }^{24}$

Questi pur occasionali rilievi possono suggerire la presenza di diversi gradi di sovrapposizione e di sfasatura, rispetto alla tipologia paradigmatica del petrarchismo, in particolare bembiano, tra asse di significato, architettura sintattica e partitura metrica. La posizione stilistica dell'A riosto rimatore, a guardar bene, potrebbe risultare ancora eccessivamente 'ridotta' per l'esigenza storico-letteraria, astratta e postuma, di inserirne l'esperienza tra $i$ due estremi della lirica cortigiana e del Bembo petrarchista. Vale la pena, forse, di provare a verificare l'ipotesi di un Ariosto che si muova parallelamente al 'compagno di strada' Bembo, che ne condivida $i$ valori imitativi di riferimento, ma abbia in mente obiettivi diversi, proponga cioè un'altra possibile via, poi storicamente perdente, del petrarchismo cinquecentesco.

Alberto Roncaccia 
I. Facciamo riferimento, in particolare, ai seguenti studi, edizioni, antologie: Stefano Bianchi (a cura di), Ludovico Ariosto, Rime, Milano, Rizzoli («BUR»), 1992; Emilio Bigi, Poesia latina e volgare nel Rinascimento italiano, Napoli, Morano, I 989 (in part. pp. I 89-227); Nino Borsellino, Ludovico Ariosto, Roma-Bari, Laterza, I 979 (in part. pp. 20-2 I); Cesare Bozzetti, in Le Rime di Ludovico Ariosto secondo il codice Rossiano (Vat. Ross. 639) nell'edizione e col commento ai testi I-XX di Cesare Bozzetti, a cura di Claudio Vela, in Fra Satire e Rime ariostesche, a cura di Claudia Berra, Milano, Cisalpino, 2000, pp. 223-3 10; Alberto Calciolari, Inquietudini settentrionali nell'Età di Bembo, in Lirici europei del Cinquecento. Ripensando la poesia del Petrarca, a cura di Gian Mario Anselmi et al., Milano, Rizzoli («BUR»), 2004 (in part. pp. 234-35); Stefano Carrai, L'usignolo di Bembo. Un'idea della lirica italiana del Rinascimento, Roma, Carocci, 2006 (in part. alle pp. 85-ıo9); Georges Güntert, Per una rivalutazione dell'Ariosto minore: le Rime, in «Lettere italiane» (1971), pp. 29-42; Roberto Fedi, La memoria della poesia. Canzonieri, lirici e libri di rime nel Rinascimento, Roma, Salerno Editrice, 1990 (in part. pp. 83-I I 5); Giulio Ferroni, Ariosto, Roma, Salerno Editrice, 2008 (in part. pp. 33-4I); Roberto Gigliucci (a cura di), La lirica rinascimentale, introduzione di Jacqueline Risset, Roma, Istituto Poligrafico e Zecca dello Stato, 2000 (in part. pp. 297-98); Stefano Jossa, Ariosto, Bologna, il Mulino, 2009 (in part. pp. I 3-I 5); Mario Santoro (a cura di), Ludovico Ariosto, Opere, vol. 3, Carmina, Rime, Satire, Erbolato, Lettere, Torino, UTET, I 989 (in part. p. 222); Cesare Segre (a cura di), Ludovico Ariosto, Opere minori, Milano-Napoli, Ricciardi, 1954 (in part. pp. I35-136).

2. Ferroni, Ariosto cit., pp. 39-40.

3. Bozzetti, Le Rime di Ludovico Ariosto secondo il codice Rossiano cit.

4. Carrai, L'usignolo di Bembo cit., p. 99.

5. Carrai, L'usignolo di Bembo cit., p. 98.

6. Bigi, Poesia latina e volgare cit., p. 209.

7. Precisa Ferroni: «È un compromesso che rappresenta una possibile strada, che avrà scarso seguito nella lirica petrarchistica: quella di una fusione 'media' tra comunicazione equilibrata e istanze del quotidiano, tra chiusura entro un circolo 'perfetto' e apertura alla varietà dell'esperienza, tra assolutezza e occasionalità» Ferroni, Ariosto cit., p. 36.

8. Osserva Borsellino: «Come pura operazione letteraria la lirica dell'Ariosto non è né un passivo riadattamento di situazioni tipiche del Canzoniere [...] né un'accettazione dei canoni, peraltro non ancora correnti, del petrarchismo cinquecentesco [...]. Proprio l'insistenza sulla schiavitù d'amore rivela il tentativo di attraversare il Petrarca per ricongiungersi a zone liriche più lontane: alla poesia cortese e allo stilnovo» Borsellino, Ludovico Ariosto cit., p. 2 I.

9. Bozzetti, Le Rime di Ludovico Ariosto secondo il codice Rossiano cit., p. 243.

ıo. Arnaldo Soldani, La sintassi del sonetto. Petrarca e il Trecento minore, Firenze, Edizioni del Galluzzo, 2009. 
i i. Per la tradizione del tema cfr. la nota di Gigliucci, La lirica rinascimentale cit., p. 298.

I 2. Sulla rappresentazione della prigionia amorosa in Petrarca e sulle sue ascendenze bibliche, in particolare per il tema del 'laqueus' amoroso come «laccio nemico di segno erotico negativo", è molto utile il capitolo Vincola amorosi del libro di Silvia Chessa, Il profumo del sacro nel canzoniere di Petrarca, Firenze, Società Editrice Fiorentina, 2005, pp. I 55-248.

I 3. L'indicazione è in Santagata, a commento dei versi citati del sonetto 349. Cfr. Marco Santagata (a cura di), Francesco Petrarca, Canzoniere, Milano, Mondadori, I996, p. I 329 .

i 4. Santoro (a cura di), Ludovico Ariosto, Opere cit., pp. 88-89. Possiamo ricordare i due distici conclusivi del componimento, di emblematica intonazione properziana: «Ah! pereat qui in amore potest rationibus uti; / ah! pereat qui ni perdite amare potest. / Quid deceat, quid non, videant quibus integra mens est; / sat mihi, sat dominam posse videre meam». Il tema della vera qualità dei rapporti amichevoli tra $\mathrm{i}$ due scrittori è riproposto recentemente da Alessandro Gnocchi, che nota come all'altezza della risposta Ad Petrum Bembum il Veneziano a Ferrara dovesse essere «più noto come figlio di Bernardo, già vicedomino della città nel I 498-I 499, che come astro nascente della letteratua» (Alessandro Gnocchi, Tommaso Giustiniani, Ludovico Ariosto e la Compagnia degli Amici, in «Studi di filologia italiana», I999, vol. LVII, pp. 277-203, a p. 289).

i s. Pietro Bembo, Rime, a cura di Guglielmo Gorni, in Poeti del Cinquecento. Tomo I. Poeti lirici, burlescbi, satirici, didascalici, a cura di Guglielmo Gorni, Massimo Danzi e Silvia Longhi, Milano-Napoli, Ricciardi, 200I, pp. 39-225, a p. 172. Per il componimento e la sua cronologia si veda l'edizione commentata di Donnini (Pietro Bembo, Le rime, a cura di Andrea Donnini, Roma, Salerno Editrice, 2008, pp. 294 e 299-300).

I6. Come ricorda Silvia Chessa, nella tradizione patristica il venator è il demonio, Chessa, Il profumo del sacro cit., p. I 56.

17. Il sintagma non è presente in Petrarca e Bembo, dove, al termine "parole», sono spesso riferite espressioni come: 'accorte e sagge', 'pietose e dolci', 'honeste e accorte', 'angeliche', 'leggiadre e care', 'alte e sante', 'soavi', etc.

I8. In Petrarca il termine ha una connotazione spirituale, se pensiamo, tra $i$ numerosi esempi, ai 'lacci sciolti' del peccato nella canzone alla Vergine, dove Cristo «nostri lacci à sciolti» (Rvf 366,49), o a quelli del corpo mortale da cui l'anima è «sciolta» in $R v f 28$, I 3, che si sovrappone a quella amorosa: "costei che 'n fuga è volta, / et de' lacci d'Amor leggiera et sciolta» $R v f$ 6, 2-3.

I9. Si può osservare la ripresa del tema in senso moraleggiante e classicheggiante in un contemporaneo di Ariosto come Renato Trivulzio, che, in una delle sue I 34 rime, riprende l'incipit di Rvf 89 variando l'ordine dei termini chiave: «Di tua prigion, Amor, dura fuggendo, / Rotti e spezzate i ceppi e le catene / Del disio folle e de la falsa spene, / Immortal' gratie al ciel lieto ne rendo, // E dipinte, 
Diana, i' ti sospendo / L'altrui fierezze al tempio, e le mie pene; / Et ornando di fiori e di verbene, / Gli odiosi mirti ne gli altar' t'accendo, // E due nere agne, hor che la notte imbruna, / T'uccido, e due bianche altre a la matina, / Cantando le tue lodi ad una ad una: // Figlia di Giove più ch'altra divina, / Proserpina in inferno, e nel ciel Luna, / Diana ne le selve unica e trina», trascritto dal ms. Ambrosiano V 24 sup., a p. I44, in Simone Albonico, Le odi di Renato Trivulzio. Edizione critica e saggio di commento, Tesi di dottorato di ricerca (IV ciclo), Pavia, I992.

20. Marco Praloran, Metrica e tecnica del verso, in Prose della volgar lingua di Pietro Bembo, a cura di Silvia Morgana, Mario Piotti, Massimo Prada, Cisalpino, Milano, 200I, pp. 409-2I.

2 I. Di questo tipo è ad esempio il verso finale della canzone alla Vergine, che chiude anche i Rvf: «ch'accolga 'l mïo spirto ultimo in pace».

22. Nota Bozzetti: «Si può aggiungere, per scrupolo, che in Petrarca, al singolare, 'giuoco' rima spesso con 'poco'» Bozzetti, Le Rime di Ludovico Ariosto secondo il codice Rossiano cit., p. 24).

23. Menichetti definisce «attacco duro» questa precisa dialefe, di cui prende esempio proprio da Ariosto, Furioso X 88, 7, Aldo Menichetti, Metrica italiana. Fondamenti metrici, prosodia, rima, Padova, Antenore, i 993, p. 347.

24. Fedi, La memoria cit., p. I04. 